\title{
EXTENSION THEOREMS FOR REDUCTIVE GROUP ACTIONS ON COMPACT KAEHLER MANIFOLDS
}

BY ANDREW J. SOMMESE

Communicated by Stephen S. Shatz, March 13, 1975

Let $G$ be a connected complex reductive Lie group. Noting [3], [7], [8] that $G$ has the structure of a linear algebraic group, let $\bar{G}$ be any projective manifold in which $G$ is Zariski open and which induces the above algebraic structure on $G$. The purpose of the present note is to announce

Proposition I. Let $G$ be as above and act holomorphically on a compact Kaehler manifold $X$. Assume that the Lie algebra of holomorphic vector fields on $X$ generated by $G$ is annihilated by every holomorphic one form. Let $\Phi: Y \rightarrow X$ be a holomorphic map where $Y$ is a normal reduced analytic space. Consider the equivariant map $\Phi^{\prime}: G \times Y \rightarrow X$; $\Phi^{\prime}$ extends meromorphically (in the sense of Remmert) to $\bar{G} \times Y$.

REMARKs. The condition on vector fields annihilated by one forms is automatically satisfied if (cf. [12]-[14]) $H^{1}(X, \mathrm{Q})=0$, or $G$ is semisimple, or if every generator of the solvable radical of $G$ has a fixed point, or if $G$ is a linear algebraic group acting algebraically on a projective $X$. Taking $Y$ to be a point, one gets the orbits of $G$ to be Zariski open in their closures which are analytic sets. A simple corollary is the classical result that there is only one structure of a linear algebraic group on $G$ (cf. [7]), and in fact any reductive connected subgroup of an algebraic group over $\mathbf{C}$ is an algebraic subgroup.

As a further application of the techniques used, a new proof of an improved form of a fixed point theorem (cf. [12], [13], [14]) of the author is given:

Proposition II. Let $S$ be a connected solvable Lie group acting holomorphically on a compact Kaehler manifold $X$. The following are equivalent:

(a) $S$ has a fixed point on $X$.

AMS (MOS) subject classifications (1970). Primary 32M05, 32J25, 53C55; Secondary $22 \mathrm{E} 10$. ic groups.

Key words and phrases. Reductive group actions, Kaehler manifolds, linear algebra- 
(b) $S$ leaves a compact set in a fibre of the Albanese map invariant.

(c) $S$ has a fixed point within any compact set $K$ on $X$ that $S$ leaves invariant.

(d) The Lie algebra of vector-fields that $S$ generates on $X$ is annihilated by every holomorphic one form on $X$.

REMARK. The assertion (c) where $K$ is any compact set is new; the method of proof allows one to relax the compactness of $X$ and show if, in addition, $H^{1}\left(X, O_{X}\right)=0$, then (c) is true.

The following is the fundamental observation on which everything rests.

Lemma. Let $X$ be a compact Kaehler manifold and $\rho: \mathrm{C}^{*} \rightarrow \operatorname{Aut}(X) a$ holomorphic $\mathrm{C}^{*}$ action that has at least one fixed point. Let $A: \mathrm{C}^{*} \rightarrow X$ be a holomorphic equivariant map onto an orbit: then $A$ extends to a homomorphic equivariant map $\widetilde{A}$ of $\mathrm{CP}^{1}$ to $X$.

Proof. Assume without loss of generality that $A\left(\mathrm{C}^{*}\right)$ is not a point. Let $\mu$ be a Kaehler metric on $X$ and $\omega$ the associated Kaehler form. Assume that $\mu$ has been averaged with respect to the circle subgroup $S^{1} \subseteq \mathrm{C}^{*}$. Let $\chi$ be the holomorphic vector-field on $X$ associated to $\rho: \mathrm{C}^{*} \rightarrow \operatorname{Aut}(X)$.

Because of equivariance, the Jacobian, $d A$, of $A$, maps some constant multiple of $z(\partial / \partial z)$ onto the restriction of the vector-field $\chi$ to $A\left(C^{*}\right)$. Without loss of generality this constant is assumed to be one.

Let $A^{*} \mu=a(r) d z \otimes d \bar{z}$ where $a(r)$ is positive and depends only on $r$ due to the $S^{1}$ averaging of $\mu . A^{*} \omega=(i / 2) a(r) d z \wedge d \bar{z}$.

$$
\begin{aligned}
\mu(\chi, \chi) & =\mu\left(d A\left(z \frac{\partial}{\partial z}\right), d A\left(z \frac{\partial}{\partial z}\right)\right)=A^{*} \mu\left(z \frac{\partial}{\partial z}, z \frac{\partial}{\partial z}\right) \\
& =a(r)|z|^{2} \leqslant M<\infty
\end{aligned}
$$

where $\sup _{X} \mu(\chi, \chi)=M<\infty$.

Now by Lichnerowicz [5] there exists a $C^{\infty}$ function $\phi$ on $X$ such that $\bar{\partial} \phi=\omega(\chi)$. Pulling back and, without confusion, letting $\phi$ stand for $A^{*} \phi=$ $\phi(A(z))$, one has

$$
\frac{i}{2} z a(r) d \bar{z}=\frac{\partial \phi}{\partial \bar{z}} d \bar{z} \quad \text { or } \quad \frac{i}{2} z a(r)=\frac{\partial \phi}{\partial \bar{z}} .
$$

Now fix one circle, say the unit circle $C_{1} \subset \mathrm{C}^{*}$ and let $C_{R}=\left\{z \in \mathrm{C}^{*} \mid\right.$ $|z|=R\}$. Assume $R>1 ; C_{1}$ and $C_{R}$ bound an annulus $\widetilde{A}$ with $\partial \widetilde{A}=C_{R}-$ $C_{1}$. Now 


$$
\begin{aligned}
\int_{\widetilde{A}} \int_{A^{*} \mu} & =\int_{\widetilde{A}} \int_{2} \frac{i}{2} a(r) d z \wedge d \bar{z}=-\int_{\widetilde{A}} \int \frac{\partial \phi}{\partial \bar{z}} \frac{d \bar{z} \wedge d z}{z} \\
& =-\int_{C_{R}} \phi \frac{d z}{z}+\int_{C_{1}} \phi \frac{d z}{z}=\frac{1}{i} \int_{0}^{2 \pi} \phi\left(R e^{i \theta}\right) d \theta-C
\end{aligned}
$$

with $C$ a constant. Now $\left|\int_{0}^{2 \pi} \phi\left(R e^{i \theta}\right) d \theta\right| \leqslant M^{\prime}<\infty$ since $\phi$ is the pullback of a bounded function on $X$.

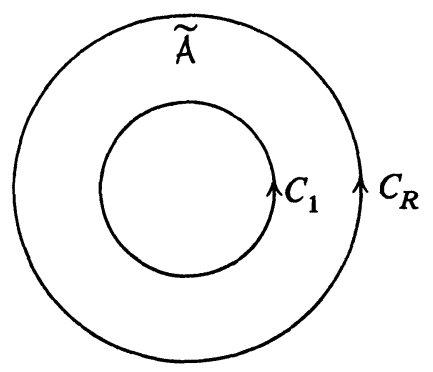

Therefore $\iint_{\tilde{A}} A^{*} \mu \leqslant M^{\prime \prime}<\infty$ where $M^{\prime \prime}$ is a positive constant independent of $R$. Thus by Bishop's extension theorem (cf. [1], [2]), $A$ extends holomorphically over $\infty$. An identical argument gives extension at 0 . Q.E.D.

Using the above Lemma and the Levi-Griffiths-Shiffman-Siu extension theorem (cf. [2], [9], [10], [11]) repeatedly, one proves the result for $\operatorname{SL}(2, \mathrm{C})$ and groups of the form $\left(\mathbf{C}^{*}\right)^{n}$ that have a fixed point on $\mathrm{X}$. Then one proves it for one parameter unipotent subgroup of $G$ by using the above $\operatorname{SL}(2, \mathrm{C})$ result on an $\operatorname{SL}(2, C)$ in $G$ containing the subgroup; this can be done by Jacobson-Morosow (cf. [4]). One now proves it for a Borel subgroup of $G$ and uses an argument depending on the fact that one has a locally trivial fibring of $G$ over $G / B$ which is compact.

In the very interesting paper [6] of Lieberman, related matters are discussed.

\section{BIBLIOGRAPHY}

1. E. Bishop, Conditions for the analyticity of certain sets, Michigan Math. J. 11 (1964), 289-304. MR 29 \#6057.

2. P. A. Griffiths, Two theorems on extensions of holomorphic mappings, Invent. Math. 14 (1971), 27-62. MR 45 \#2202.

3. G. Hochschild and G. D. Mostow, Automorphisms of affine algebraic groups, J. Algebra 13 (1969), 535-543. MR 41 \#315. 
4. N. Jacobson, Lie algebras, Interscience Tracts in Pure and Appl. Math., no. 10, Interscience, New York and London, 1962. MR 26 \#1345.

5. A. Lichnerowicz, Variétés kähleriennes et première classe de Chern, Differential Geometry 1 (1967), 195-223. MR 37 \#2150.

6. D. Lieberman, Holomorphic vector-fields and rationality (unpublished manuscript).

7. G. D. Mostow, Representative functions on a Lie group, Some Recent Advances in the Basic Sciences, Vol. 2 (Proc. Annual Sci. Conf., Belfer Grad. School Sci., Yeshiva Univ., New York, 1965-1966), Belfer Graduate School of Science, Yeshiva University, New York, 1969, pp. 209-226. MR 42 \#6156.

8. J.-P. Serre, Algèbres de Lie semi-simples complexes, Benjamin, New York and Amsterdam, 1966. MR 35 \#6721.

9. B. Shiffman, Extensions of positive line bundles and meromorphic maps, Invent. Math. 15 (1972), 332-347.

10. Y.-T. Siu, Analyticity of sets associated to Lelong numbers and the extension of meromorphic maps, Bull. Amer. Math. Soc. 79 (1973), 1200-1205.

11. - Analyticity of sets associated to Lelong numbers and the extension of closed positive currents, Invent. Math. 27 (1974), 53-156.

12. A. J. Sommese, Algebraic properties of the period mapping, Thesis, Princeton Univ., 1973.

13. - Borel's fixed point theorem for Kaehler manifolds and an application, Proc. Amer. Math. Soc. 41 (1973), 51-54. MR 48 \#579.

14. Holomorphic vector-fields on compact Kaehler manifolds, Math. Ann. 210 (1974), $75-82$.

DEPARTMENT OF MATHEMATICS, YALE UNIVERSITY, NEW HAVEN, CONNECTICUT 06520 